\title{
Using technology in business education
}

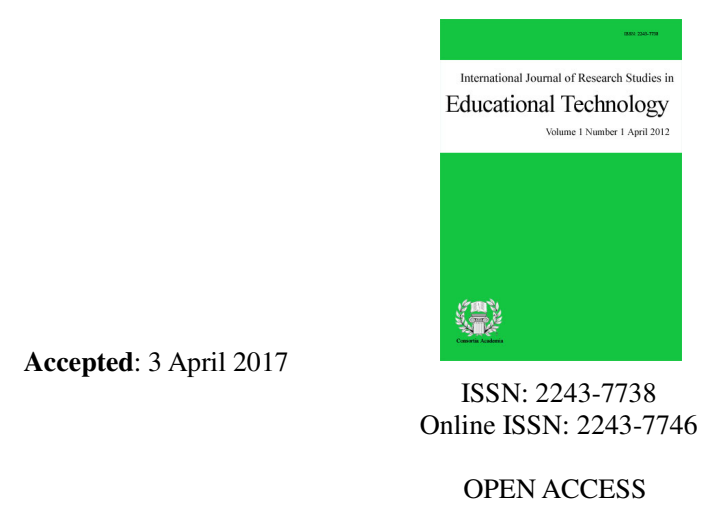

\section{Abstract}

Online education, hybrid courses, and other types of distance learning opportunities continue to revolutionize and change how institutions, faculty, and learners approach and evaluate higher education options. While the technological options, including the proliferation of social media tools, can appear to be overwhelming at first, the method is less important than the information distributed via online platforms. Appealing both to current students, especially millennials and Gen-Z individuals, and adult learners that are returning to college while also working, online options provide both challenges and opportunities. Colleges and universities, from administrators, to faculty, to staff must be aware of both the options for higher education platforms and how to construct appropriate content. The decisions made surrounding course engagement, course content, communicating and interacting with students, and building an inclusive environment are essential to the successful implementation of online courses and program. In a continuously changing environment such as online learning and education, a logical approach, instead of attempting to construct a permanent solution from the beginning, is to take into account the various factors and forces that can harnessed to drive positive change. This piece reviews current research, discusses implications for members of the higher education community, and outlines a checklist for practices and ideas to take into account as online learning continues to drive innovation.

Keywords: innovation; online learning; technology; accounting 


\section{Using technology in business education}

\section{Introduction}

Technology continues to revolutionize and dramatically change the higher education landscape, but there remains a continuing struggle and confusion as to how best effectively integrate technology into quantitative business education. Analyzing both media coverage and academic research it is readily apparent that technology is embedded within virtually every aspect of society and business. Online education, hybrid courses, and other distance learning options are gradually, and increasingly at rapid pace, changing the landscape of higher education (Kentor, 2015). In spite of these advances, however, developing and implementing effective and high quality online education courses remains an ongoing challenge for faculty and institutions. Compounding this challenge is the reality that incoming students, specifically millennials and members of Gen-Z, are digital natives, and are of the mindset that technology should be embedded within the educational process. This assumption and expectation provide both opportunities and challenges for the faculty and institutions seeking to attract these students. Additionally, and perhaps unexpectedly, another cohort of incoming students is attracted to the appeal of online learning, and increased integration of technology in the learning process. Adult learners, either already within the workforce or in transition between various positions, are usually trying to juggle personal lives and professional obligations while also returning to school to learn new skills or refresh existing ones. Framed in this light the following reality facing online education begins to crystallize; online education and remote learning are increasingly the trends that will be influencing higher education. Developing quality courses, training faculty and staff to develop and implement online learning opportunities, and embedding online education into the fabric of the institution are requirements that will only grow in importance moving forward.

\subsection{Technology options}

Various technology options exist, not only for social and individual utilization but also the creation and implementation of effective online strategies. Regardless of the technology options selected, there is a distinct emphasis on the content used to support the course design and instructional tactics of instructors (Kramer, Neugebauer, Magenheim, \& Huppertz, 2015). Clearly, in order to compensate for the reduction in interaction there has to some alternative to the traditional textbook based platform used for in-person instruction. Depending on the institution in question, faculty preferences and opinions, and the various procurement agreements in place for this and other items the individual learning management system (LMS) might very well change or be different for various departments or schools at the university. Pricing, features, and compatibility with existing information technology infrastructure all are driving forces in deciding which LMS to implement and build out, but there are several key factors that differentiate higher quality options from other options. The list below is by no means meant to be exhaustive, but may be viewed as illustrative of common features and functionality that should be integrated into the decision making process.

$>$ Compatibility - Making sure that the various LMS platforms are compatible and able to be integrated within the broader technology infrastructure is essential. For example, students should be able to access institutional resources such as the library and other staff resources from the LMS used for online classes.

$>\quad$ Ease of use - Especially for hybrid or fully online classes, the technology selected for utilization should be easy to use, updated consistently, and able to integrated with third party platforms. Textbook and publishing organizations, for example, have created online content independent of universities -this content should be embedded in the online learning experience. 
$>$ Support - Arguably the most important part of the online education process is to institute support for these initiatives at an institutional level, from staff, faculty, and administrators. Whether it involves training, financial resources, and marketing the program effectively, support for these new courses and ideas must consistent and embedded into the college or university.

\section{Building an online environment}

Perhaps the most important part of building and delivering a quality online environment is to identify the core components of the online course that would form the backbone of the digital learning experience. It is also important, linking back to the different student groups institutions seek to attract, the importance of culture and different competencies such different groups bring to the proverbial table (Gomez-Rey, Barbara, \& Fernandez-Navarro, 2016). For example, certain students might benefit more from audio content versus other students more attracted to text based learning - these factors should be considered as online environments are constructed. Understanding that many of the students seeking out online education are either digital natives, or interested in leveraging online learning to effectively manage time provides an opportunity for course development and implementation. Subsequent to the selection of a learning management system the next step to undertake is the construction and building out course content and objectives. Selecting effective objectives, including a variety of measures to evaluate students, and providing an open and inclusive environment for discussion and productive debates are essential components of the learning experience. Drilling specifically into some of the various aspects of the online learning that should ideally take precedence, the following criteria and objectives appear to logical and reasonable.

First, a variety of measures should be integrated into the course shell, regardless of which platform is utilized. Included in this shell construction and development should be options for content to be uploaded the instructor, a forum for students to interact and engage with each other, an assignments section, and instructor uploaded content. Building an effective environment for students to engage with each other, and interact with both fellow students and the instructor, should, is an important feature for online learning initiatives (Jian Ping, 2016). Second, an announcement page must be integrated into the course shell to allow the instructor to provide updates and other types of information to students throughout the semester. Third, and perhaps most importantly to the smooth running and management of a fully online course is the ability of the instructor and students to communicate in a timely manner. For example, an effective method of establishing this protocol might be to outline email policy in the syllabus, and state that all emails will be responded to within a certain time frame (24 - 48 hours might be a serviceable example).

Drilling in specifically to the idea of content uploaded to the course shell, and instructor created content, it is important that a certain balance be reached between these two sources. Professionally created and edited content, clearly, can add significant value to the learning experience and is on par with expectations from students expecting a technologically integrated learning experience. That said, it is also important to recognize the fact that, by adding original content, even if it is just a voice over recording of a PowerPoint lecture, can add a dimension to the course that might otherwise be lacking. Especially important in a virtual environment, it is important for both students and instructors to feel that the learning experience is comprehensive in nature. While multiple tools exist to upload content into course shells, the specific tool that it utilized is less important than the fact that faculty in charge of online learning and course development be well versed in the utilization of technology.

\subsection{Assessments}

Assignments, and learning assessments, provide both a challenge and opportunity to faculty and institutions seeking to develop a robust and comprehensive online platform. In addition to providing both students and instructors with information, increased can be formatted in reports and dashboards to help assess the effectiveness of various learning strategies (Hogaboam, Chen, Hmelo-Silver, Lajoie, Bodnar, Kazemitabar, \& 
Chan, 2016). Whether the course content is taught in person or in a virtual environment, it is imperative that the following truth remain constant - students must be given an opportunity to prove their understanding of the content. Of critical importance is the understanding that not every student is equally as adept at taking exams, writing reports and analyses, or giving presentations. Providing a variety of options that allow students to demonstrate knowledge and understanding of the content taught in the course is an essential part of building an effective online pedagogy. Especially for quantitative courses including accounting, finance, and economics, where the technical course content can be challenging for the best students, developing a robust assessment program is an important step in course design.

When it comes to online teaching, it is important to make efficient use of the technology that is available, and this links back to using an LMS that is compatible with third party platforms and information. Instead of having to create, from scratch, every question for every assignment or exam, instructors and faculty should leverage existing content made available by textbook publishers. This content may include, but is not limited to question banks, Excel scenarios, exam banks, video lectures, and additional PowerPoint resources. In addition to helping ensure a consistent tone and type of question that are asked, making use of these available resources allows the faculty to focus on higher level tasks such as research, publishing, and communicating the value of the program across academic channels. This leads to the next aspect of online education that should emphasized, and will be of benefit to both students and faculty involved.

\subsection{Engagement}

Engagement and retention of students, whether they are traditional or non-traditional students, is of paramount importance to both institutions and the students enrolled at the institution. The issue of student debt, which continues to be a high profile issue for both political and financial markets, has a secondary side that must be discussed. Student debt can be considered in the education of the individual, but also whether or not the debt is incurred by students that finish undergraduate curriculum. Building a robust and engaging online learning community and environment is imperative to keeping online learners enrolled and engaged throughout the entirety of the education process. While the idea of immersive or completely interactive classrooms may still yet remain out of reach for most institutions, interaction is essential to assist in enhancing the online learning experience (Andrew, Maslin-Prothero, \& Ewens, 2015). Keeping students engaged, however, is only half of the proverbial struggle to boost and maintain enrollment. In the face of increased competition, rising pressure on costs, and a focus on outcomes for students, online education and learning options provides an opportunity to assist with student retention.

Retention is an important force in the higher education industry, pointedly because it is a component in several key rankings and assessments related to institutional competitiveness. Attractiveness to parents and potential students, retaining current students, and helping to build a culture of academic success and completion represent areas that all administrators and faculty must take into account. Lastly, but perhaps most importantly regarding the conversation around online education and distance learning, is the flexibility that establishing an online program provides to students. Retaining students, particularly in a climate focusing on college affordability, student success, and educational outcomes, the ability to engage with students in a meaningful and continuous manner is essential.

\section{Content and Training}

Arguably the most challenging aspect of developing, implementing, and successfully maintaining a robust online learning environment is ensuring a worthwhile blend of content. Discussed above, the research and market evidence indicates that a combination of third party, or professionally generated, content, and content that is instructor created provides a unique blend of engagement and information. Such a combination or blend of content, of course, sounds ideal in the realm of high level planning and strategy, but the day to day implementation of such a strategy can seem overwhelming. Especially when confronted with the proliferation of 
technology, including mobile based apps and learning tools, it is tempting to simply upload an extremely large amount of information instead of focusing on the quality of the information. Doing so, however, runs the following risk - by uploading too much information, or using too many different options, can lead to students tuning out most of the information anyway. Framed against this backdrop, and particularly when taking into account the competition of YouTube, Facebook, and other social media channels, the critical point is that the content included in online learning must be targeted and meaningful.

For example, and linking back to the idea of providing a variety of opportunities for students to demonstrate knowledge of course materials, a variety of content creates a platform agreeable for students regardless of learning preference. Discussion forums, written assignments, questions provided either from the textbook or online course content, written assignments and reports, and traditional exams are also readily able to be uploaded in an online course shell. Obviously, the specifics of the content, and the frequency of certain types of information and content will differ from course to course, and instructor to instructor, but the underlying concept is the same. For example, research reinforces the reality that some students will learn more effectively via audio materials, whereas others will benefit from text based information (Oliveira Neto, Huang, \& Azevedo Melli, 2015). Building a satisfactory online platform and courses requires both effective content and individuals that are well versed in utilizing technology in the learning experience. Training, both upfront and ongoing, is certainly required for individuals seek to leverage online learning, even for traditionally quantitative courses such as finance and accounting. While courses in corporate communication and investor relations might use more sophisticated video technologies, for example, quantitative courses will certainly benefit from better utilization of technology.

\subsection{Staff training}

The foundation and backbone of any initiative must include training and educating the staff that will support both the student and faculty users of the program. Onboarding new members to the information technology team, expanding the skill sets of existing employees, and making more resources available form the basis for a quality training program. It is also important for the administrators and upper level staff members to create a culture that is conducive to building and maintaining online programs. Support and enthusiasm for online learning and technology can take the form of high level training, mission statements, and most importantly, continuous funding and updating. Investing in the training and development of staff level competencies, as they pertain to online learning and technology tools, represents an investment that will benefit both students and faculty. Simply put, without the proper support to be able to address the inevitable questions and concerns that will arise with technology, neither students nor faculty will reap the fully benefits achievable by using such technology.

\subsection{Student guidelines}

Students, particularly first time students new to the college experience, enter the learning environment with familiarity with digital technology, tools, and how to interact in a virtual landscape. That said, and specifically with the issues of cyber bullying and harassment looming large on college campuses, specific guidelines must be established to assist in creating an inclusive and welcoming online space for students to learn and develop within. In addition to specifying desired discussion posts lengths and response criteria, both the institution and faculty tasked with developing online courses must be vigilant to reinforce the ideas of civility, decorum and mutual respect in online classes. The virtual classroom is still a classroom, and it must be an environment that both students and faculty feel comfortable sharing ideas, opinions, and conducting debates and conversations in a respectful tone. Such a challenge provides an opportunity for administrators at the institution to assume a leadership position to work with faculty and student groups to solicit feedback, develop beta programs for testing, and fine tune these guidelines as needed. Sometimes overlooked, due to an assumption that many traditional college age students already familiar with using technology, establishing criteria for using technology for academic purposes is an important step in the development process. 
As digitally savvy as incoming traditional age college students may be, there is a distinct percentage of faculty members that may not be as well versed in using technology for online or hybrid instruction. Fortunately, there are several methods by which this gap can be addressed, least of all the reality that many of the leading LMS tools are increasingly user friendly and intuitive to use. Additionally, and building on the user friendly nature of online learning tools, is the increasing integration of technology into the everyday life; this makes the transition and learning curve less dramatic, and facilitates the adoption of online tools amongst the ranks of faculty. Specifically, and linking back to the importance of staff training and the availability of institutional resources, in-person and hands on training can be used to great effect to help facilitate the adoption of these tools. On top of the physical sessions, however, faculty should be encouraged to test online access and usability of tools from off-site locations, i.e., faculty workspaces to ensure connectivity and access are not impacted.

\section{Course Selection and Technology}

Addressing the positives and negatives of online learning and education it is possible to assume all courses can be transitioned from live, or in-person sessions, to those conducted entirely online. Specifically, the increasing utilization of social media by students, faculty, and institutions alike makes attempts to develop a social network to assist in online learning all the more relevant to monitor and evaluate moving forward (Kon Shing Kenneth, \& Paredes, 2015). While still in early stages, clearly, developing a social first online learning model might be of benefit, specifically for qualitative courses. Drilling in deeper, however, it readily becomes apparent that, at the present, courses such as public speaking, drama, or course aligned with the arts or expressive content are not readily applicable to be taught in an online format or in an online learning environment. Quantitative courses, including both STEM and business courses, however, can and should be explored and tested for adaptation to hybrid or online instruction. Accounting, finance, management, and economics form the backbone of how business managers make decisions in the marketplace, and it is essential that business schools educate students in these competencies. Arguably more important, for the purposes of building and implementing an online learning platform is making sure that technology is built into the course experience. Simply uploading a syllabus, assignments, and various types of information is insufficient - the course instruction and other information must also teach students how to use technology for business purposes.

The specific tools will, of course, vary from institution to institution and be different depending on the types of courses that are being taught, but there are several traits and characteristics that are important for consideration. One example of information and technology that should be studied and evaluated are MOOC's and the effects and changes they are bringing to the higher education industry (de Freitas, Morgan, \& Gibson, 2015). First, the technology used, such as Adobe Connect or Skype, for example, should be a widely adopted program that is routinely updated and maintained by an institutional program. In other words, as helpful as Wikipedia might be for satisfying cursory searches, such an open sourced program might not be an ideal base from which to build an online learning environment. After the specific tool has been selected, an equally important part of developing an online environment comes into play - the striving for continuous improvement. Updating, tweaking, and changing the specifics of how certain programs can be used reflects how quickly technology changes in the broader market at large. Linking these changes to the changes enacted within the course room provides the dual benefit of better technology and a more realistic approach to using technology for business purposes.

An ancillary benefit to using technology in the digital classroom, however, is to also promote opportunities for additional publishing, both in academic circles and in more practitioner based publications. For faculty, tenure track and non-tenure track, can benefit from additional publishing and exposure opportunities, as well as using technology to seek out and obtain speaking activities and opportunities. Faculty should especially be interested in leveraging the online classroom and environment to further publishing and speaking, as both of these form the cornerstone of scholarship activity. There is, however, an additional aspect that should be taken 
into account, and this the opportunity for greater student publishing and outside activities. Students, especially in the increasingly competitive environment and job market, continuously seek out opportunities to differentiate themselves from the potential competition and other candidates. Specifically, in the quantitative areas, having a track record of research, publication, and involvement within the profession will only aid in attracting potential employers.

The most important aspect of using technology does not have to do with a specific technological tool or aspect of the program itself, but rather the importance of starting to use technology and build out online course rooms in general. Obtaining continuous feedback and information from all parties involved, students, professors, and the instructors tasked with building out online courses can provide valuable insights that assist in improving the offering moving forward (Pearcy, 2014). The course itself does not have to be perfect at the very beginning, but the course offering must be available to help garner interest and enthusiasm for the online course offerings. Much like beta products and services are often tested by for profit entities in the market, it makes sense for institutions to develop various forms of products and services to evaluate engagement and success of the early stage online programs.

\section{Developing Online Courses}

Subsequent to considering the need for training, certain technical aspects that should be embedded within the technological platform, need for training, and the importance of institutional support for these initiatives, preparing a checklist or list of best practice represents a logical next step. Observing best practices and ideas used at leading institutions in online education is, of course, one path that can assist institutions in developing online programs, but it is also important to develop organic ideas and concepts unique to the institution. While developing this roadmap, or checklist, of ideas and concepts that will be used to develop and implement online learning courses and platforms, administrators and faculty should not hesitate to look outside of what would be considered normal peers. For example, a traditional liberal arts college might be able to gain insights from a primarily online based university, and vice versa. The critical component to understand when evaluating which practices and procedures to implement is to understand what the primary goals of developing online courses are to the institution and faculty.

Whether viewed from the perspective of faculty seeking to better engage students, and seeking out additional venues for publication and research, or administrators trying to increase student retention, several underlying patterns and forces are consistent. First, the online course offerings must consist of course and topics that are able to be taught online - again courses such as drama or public speaking are not well suited to be taught online. Second, training for both staff and faculty is essential to help in the actual implementation of various online course offerings. Putting together a high level strategy related to online courses and course content is, in and of itself, insufficient; the staff and faculty involved must be able to effectively utilize the tools and technology in use at the institution. Third, and linking directly to the marketing and institutional support for online courses and course content, is how these courses are marketing and targeted to potential students. Online education appeals to two distinct sets of potential students; returning adult learners and the recent cohorts of students that include both millennials and Gen-Z learners. Appealing to these two distinct, yet equally important, potential student groups should form the cornerstone of the planning, development, and implementation of online learning and courses.

\subsection{The checklist}

Like any other institutional objective, plan, or goal, in order to effectively allocate resources, personnel, and financial capital, a plan or checklist must be assembled. While it is logical to put together broad based frameworks or milestones related to the development of a project, including online education, more detail and granularity is required. A short list of goals, objectives, factors to consider, key questions, and guidelines will assist in the successful implementation of any large scale project. The specific ideas and concepts will, clearly, 
be distinct and vary from institution to institution, but there are commonalities that should be considered regardless of which institution is in question. Such a list as the one below is not meant to exhaustive or exclusive, but rather to serve as a framework from which administrators, faculty, and staff can set benchmarks and track the completion of this initiative.

$>\quad$ Set parameters - Whether the parameter in question have to do with the types of course taught online, faculty tasked with developing online content, or even the LMS utilized at the institution, setting such guidelines is critical. For example, and this question is most applicable to administrators and senior leadership - what schools of study will be at the forefront of the online initiative? Selecting the appropriate school, and with it faculty and staff support, is an essential first step that can play a key role in determining the success of failure of this initiative.

$>\quad$ Selection and training - In addition to selecting the school, whether it be engineering or school of business, the specific LMS to be used at the institution (which may involve user testing and training by itself), and what continuous training will be used going forward. Due to the very nature of online teaching and learning tools, updating and keeping the most current version in use at the institution, a training schedule and paradigm must be implemented. Training, as mentioned above, might either occur with an in-person format or virtually - the critical point is that this training must occur on an ongoing basis.

$>\quad$ Content creation and distribution - Content for online courses forms the cornerstone of how students learn and receive content online, so developing quality content and information is critical to any online education and training program. Selecting what third party platforms to use, with regards to textbooks and associated online content, or faculty created content and information is important. Deciding how much, what kind, and how often original content should be created and communicated via the online course shell is something that must be organized and planned in a logical manner.

> Marketing and student engagement - Marketing the online courses and, perhaps, entire academic programs, is something that must have resources and personnel dedicated to the process. Drilling down specifically it is important to identify groups of students, both traditional age and adult learners, should be a task that is allocated equally to both marketing staff and administrators. The best content and curriculum in the world by itself is insufficient - students must be attracted to the program in order to create a sustainable online learning environment.

$>$ Follow through - developing and sustaining an online program, individual courses, or an entire program in general requires that administrators, faculty, and staff follow up and follow through and support over time. This is arguably the most important aspect of instituting and developing a new online program, or programs, which involves an investment of time, financial resources, and personnel. From senior administration through to the newest staff members, support and dedication the online programs and initiatives must be a priority to best ensure an effective roll out and implementation.

\section{Conclusion}

Building an online program, various courses, or perhaps even an entire online program or school requires a rethinking of the roles and purposes and administrators, faculty members, and staff. In the face of these challenges, however, there are also various opportunities for faculty and staff to learn new skills, develop new competencies, and embrace the changes that are revolutionizing the higher education landscape. Students, both traditional age learners entering college for the first time and returning adult learners, are equally interested in online, hybrid, and various other distance learning options. Developing and implementing an online program and course content is a multifaceted process, clearly, but there are resources and tools available to proactive individuals seeking to take advantage of both the opportunities and challenges that online learning provides. The tools, techniques, and informational resources are available for institutions, faculty, and staff that are willing to 
embrace the shifting paradigms of higher education - the critical point is to begin the process.

\section{References}

Andrew, L., Maslin-Prothero, S., \& Ewens, B. (2015). Enhancing the online learning experience using virtual interactive classrooms. Australian Journal of Advanced Nursing, 32(4), 22-31.

de Freitas, S. I., Morgan, J., \& Gibson, D. (2015). Will MOOCs transform learning and teaching in higher education? Engagement and course retention in online learning provision. British Journal of Educational Technology, 46(3), 455-471. https://doi.org/10.1111/bjet.12268

Gomez-Rey, P., Barbara, E., \& Fernández-Navarro, F. (2016). The impact of cultural dimensions on online learning. Journal of Educational Technology \& Society, 19(4), 225-238.

Hogaboam, P. T., Chen, Y., Hmelo-Silver, C. E., Lajoie, S. P., Bodnar, S., Kazemitabar, M., \& ... Chan, L. K. (2016). Data dashboard to support facilitating online problem-based learning. Quarterly Review of Distance Education, 17(3), 75-91.

Jian Ping, J. (2016). The effect of a blended collaborative learning environment in a small private online course (SPOC): A comparison with a lecture course. Journal of Baltic Science Education, 15(2), 194-203.

Kentor, H. E. (2015). Distance education and the evolution of online learning in the United States. Curriculum \& Teaching Dialogue, 17(1/2), 21-34.

Kon Shing Kenneth, C., \& Paredes, W. C. (2015). Towards a social networks model for online learning \& performance. Journal of Educational Technology \& Society, 18(3), 240-253.

Krämer, B. J., Neugebauer, J., Magenheim, J., \& Huppertz, H. (2015). New ways of learning: Comparing the effectiveness of interactive online media in distance education with the European textbook tradition. British Journal of Educational Technology, 46(5), 965-971. https://doi.org/10.1111/bjet.12301

Oliveira Neto, J., Huang, W., \& Azevedo Melli, N. (2015). Online learning: audio or text? Educational Technology Research \& Development, 63(4), 555-573. https://doi.org/10.1007/s11423-015-9392-7

Pearcy, M. (2014). Student, teacher, professor: Three perspectives on online education. History Teacher, 47(2), $169-185$. 